\title{
Mathematical Model and Stability Analysis of Fluttering and Autorotation of an Articulated Plate into a Flow
}

\author{
Ali Bakhshandeh Rostami, Antonio Carlos Fernandes \\ Department of Ocean engineering, Federal University of Rio de Janeiro (UFRJ), Rio de Janeiro, \\ Brazil
}

\begin{abstract}
This paper is dedicated to develop a mathematical model that can simulate nonlinear phenomena of a hinged plate which places into the fluid flow (1 DOF). These phenomena are fluttering (oscillation motion), autorotation (continuous rotation) and chaotic motion (combination of fluttering and autorotation). Two mathematical models are developed for 1 DOF problem using two eminent mathematical models which had been proposed for falling plates (3 DOF). The procedures of developing these models are elaborated and then these results are compared to experimental data. The best model in the simulation of the phenomena is chosen for stability and bifurcation analysis. Based on these analyses, this model shows a transcritical bifurcation and as a result, the stability diagram and threshold are presented. Moreover, an analytical expression is given for finding the boundary of bifurcation from the fluttering to the autorotation.
\end{abstract}

\section{INTRODUCTION}

Studies on fluid-structure interaction have focused to a large extent on vibrational motions induced by the fluid flow such as fluttering [1], buffeting [2] and galloping [4]. In contrast, an appropriate attention has not been given to flow induced rotation. However, some of these flow induced rotational phenomena are researched to extract the energy from a current such as the galloping phenomenon ([3] to [7]), Buffeting phenomenon [2] or by the autorotation phenomenon ([1] and [8] to [10]). Fluttering is named for an oscillatory motion of a body exposed to fluid stream whereas autorotation is given to it continuous rotation [1]. The phenomena of the fluttering and tumbling happen for airplane wings [11], [12] and also play an important role in the modeling of some others low and high Reynolds number flows. For example, the motion of free falling paper and the flight of plate type windborne debris include these phenomena.

Previously, analytical and theoretical models for the plate tumbling motion made by Helmholtz, Kelvin, and others have focused on the two-dimensional (2D) case [13]. In addition, Pandula et al [14] investigated behavior of thin disc falling freely in viscous fluid in two dimensions. The stability analysis revealed three regions for motion of disc as stable autorotation (tumbling), rocking motion (flutter) and chaotic motion. Also, Copeland [16] supposed that autorotation of a flat plate obey planar-pendulum model which has capable oscillation and rotation. He has analyzed global bifurcations of this system by perturbation method and found that the stability of system follows saddle node bifurcation. In the similar work, Borisov et al [15] considered the motion of an elliptical body falling into an incompressible viscous fluid. They used the least square method to obtain the ordinary differential equations from processing data obtained numerically from integration of the 
Navier-Stocks equations. The comparison between the phase diagrams obtained by this solution and also by Navier-Stocks equation shows good agreement.

Parametric analysis of the behavior of an aerodynamic pendulum was done by Klimina et al [17] in order to study of a small wind power generator with a vertical axis. They proposed a mathematical model for free rotation mode of the pendulum and stability analysis was carried out on this model to find stable rotational region. Moreover, they have used the mathematical model to estimate the power extraction and as a result, they found an optimum value of extractable power by such a pendulum.

Lugt [18] solved the Navier-Stokes equations for the flow around a two-dimensional thin elliptical cylinder for different spin parameter, $S=\omega \cdot 1 / 2 U_{\text {rel }}$. He found the spin parameter at the point of stable autorotation $\mathrm{S}_{0}$, of 0.45. Moreover, Andersen et al [19] studied the dynamics of falling of a rigid card in air by numerical simulations of the two-dimensional Navier-Stokes equation and using a fluid force model based on ordinary differential equations. They have analyzed the transition between fluttering and tumbling in the ODE model and found that the period of oscillation diverges logarithmically at the bifurcation point.

On the other hand, Belmonte et al [20] conducted several experiments for investigating of fluttering and tumbling of the falling thin flat strip through the different fluids. Finally, they have proposed a phenomenological model including the inertial drag and lift which reproduces these motions.

Some other studies such as Skew [21], Iversen [22] and Smith [23] experimentally investigated autorotation of the plate which is restricted to a fixed axis perpendicular to the flow that it is physically a single degree of freedom problem. They have reported that the autorotation of vertically hinged plate is mainly dependent on; flow Reynolds number, plate aspect ratio, plate mass moment of inertia and initial angle of attack.

The main goal of this study is to derive and probe into the mathematical models which properly model different modes of rotation of a 1 DOF object through the flow. These modes include three eminent phenomena of fluttering (oscillation rotation), autorotation (continuous rotation), and chaotic rotation. Such mathematical models are reproduced from 3 DOF equations by which the motion of falling objects into the fluid are modeled.

This article at first will discuss on two well-known mathematical models which have been reported to model the motion of falling objects in 3 DOF. In the next step, these models will be reformed for $1 \mathrm{DOF}$ problem (an articulated plate in the flow) using some assumptions and techniques. Then, the results of the models will compare with experimental data which have been conducted on laboratory of wave and current (LOC) at the Federal University of Rio de Janeiro (UFRJ). At the end, dynamics of fluttering and autorotation and their bifurcation are considered.

\section{Analytical model}

\subsection{DOF Theoretical Models}


The dynamics of a card falling into resisting medium (such as air and water) is one of the classical problems in hydrodynamics and aerodynamics. Two eminent mathematical models have been reported by Belmonte-Eisenberg-Moses [20] and Andersen-Pesavento-Wang [19] for analyzing the dynamic of falling card which is 3DOF problem. Using assumptions and techniques, these 3 DOF models are used to develop the equation for which an articulated plate in $1 \mathrm{DOF}$ rotates freely into the current.

\subsubsection{Belmonte-Eisenberg-Moses Model}

One of the famous mathematical models used for falling object was reported by BelmonteEisenberg-Moses [20]. As said in former section, this phenomenological model has emerged by the results of several experiments carried out on the thin flat strip falling in the different fluids. Two types of forces which contribute in this model consist of the drag force due to the moving object in the resisting medium and the lift force due to nonzero circulation of the velocity field in the contour enclosed the body. The lift force is found by Joukowsky theorem which is formulated as $L=\rho V \Gamma$ where $\Gamma$ is the total circulation around the body. According to Kelvin theorem (i.e. conservation of circulation), the circulation remains constant in time [24]. Belmonte et al defined the circulation in their mathematical model as:

$$
\Gamma=4 \pi V \sin \varphi
$$

As can be seen from equation 1, the circulation is determined only the translational effect while, the rotational effect has been ignored. Moreover, Belmonte et al [20] stated based on their experiments that the drag forces of translational and rotational motions are depended on quadratic velocity. The forces are defined in the body coordinate system ( $\mathrm{x}-\mathrm{y}$ system) as follow:

$$
F_{x}=-\frac{1}{4} \delta_{\|} \rho C V v_{x}, \quad F_{y}=-\frac{1}{4} \delta_{\perp} \rho C V v_{y}, \quad F_{\varphi}=-\frac{1}{4} \delta_{\omega} \rho C^{4}|\dot{\varphi}| \dot{\varphi}
$$

where $\mathrm{x}$ is alongside the chord and $\mathrm{y}$ is perpendicular to the chord. The original form of the equation (hereinafter so-called Belmonte model) was reported in the global coordinate system (X-Y system of Figure 1). Nevertheless, for doing further analysis on this equation, it is appropriate to rewrite the equation in the coordinate system attached to the body $(x-y$ system of Figure 1). The dimensional form of the Belmonte model in the attached coordinate system and also in absence of gravity is given by equation 3 .

$$
\begin{aligned}
& \dot{v}_{x}-v_{y} \dot{\varphi}=\frac{1}{C F r^{2}}\left[-\delta_{\|} V v_{x}+4 \pi v_{y}^{2} \operatorname{Sgn}\left(v_{x}\right)\right] \\
& \dot{v}_{y}+v_{x} \dot{\varphi}=\frac{1}{C F r^{2}}\left[-\delta_{\perp} V v_{y}-4 \pi v_{x} v_{y} \operatorname{Sgn}\left(v_{x}\right)\right] \\
& \ddot{\varphi}=-12 \frac{\delta_{\omega}|\dot{\varphi}| \dot{\varphi}}{F r^{2}}-\frac{12 \pi}{C^{2} F r^{2}} v_{x} v_{y}
\end{aligned}
$$

where dot symbol denotes time derivative, $V=\sqrt{v_{x}^{2}+v_{y}^{2}}$, Fr is Froude number which is defined as $\mathrm{Fr}=\sqrt{M / \rho c^{3}}$ [20], $\mathrm{C}$ is chord length, and $\mathrm{M}$ is the mass of the plate. In this 
equation, Sgn represents the sign function. In these equations, the parameters of $v_{x}, v_{y}, \dot{v}_{x}, \dot{v}_{y}$ represent the velocities and accelerations parallel and perpendicular to the chord, respectively. The value of coefficients determined by Belmonte et al as:

$$
\delta_{\|}=3.52, \quad \delta_{\perp}=16.4, \quad \delta_{\omega}=0.2696
$$

It is worthy to note that this model takes into account neither added moment of inertia nor a correct expression for the circulation by ignoring the effect of rotational motion [25].

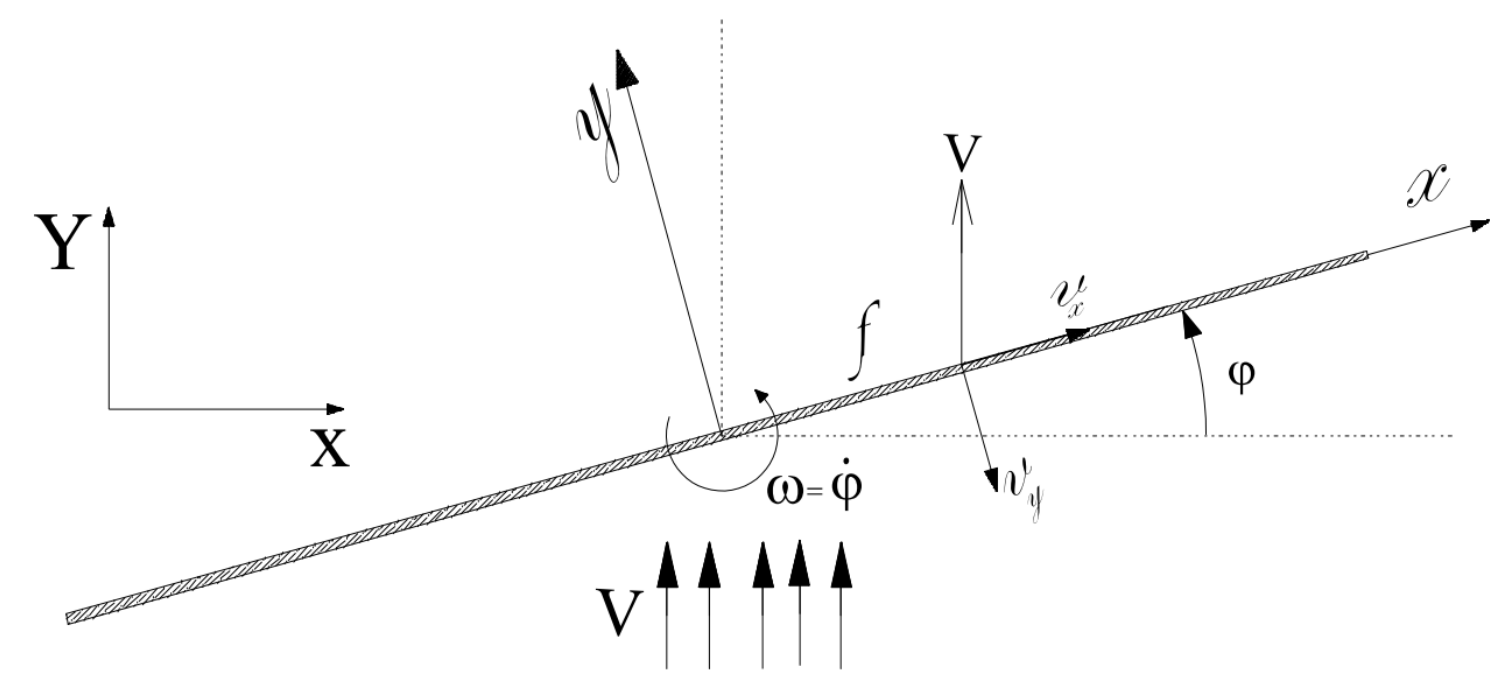

Figure 1: Schematic of the flat plate and coordinate systems that used for developing the mathematical model of 1DOF. The similar coordinate was used by Belmonte et al [20] and also Andersen et al [19].

\subsubsection{Andersen-Pesavento-Wang Model}

Another model which considers the problem of falling object has been presented by Andersen-Pesavento-Wang [19]. This model (henceforth called Andersen model) is comprised of more effective terms for analyzing the motion of an elliptic object in comparison with the Belmonte model. Andersen model takes into account not only the added mass and moment of inertia but also used a more accurate model for the circulation. The circulation is formulated by combination of the translational and the rotational terms as follow:

$$
\Gamma= \pm C_{T} C \sqrt{v_{x}^{2}+v_{y}^{2}} \sin 2 \varphi+2 C_{R} C^{2} \dot{\varphi}
$$

They have also used the quadratic terms for the lift and drag forces. Andersen model in the attached coordinate system ( $x$ - $y$ system of Figure 1$)$ is given below.

$$
\begin{aligned}
& \left(m+m_{x}\right) \dot{v}_{x}-\left(m+m_{y}\right) v_{y} \dot{\varphi}=-\rho \Gamma v_{y}-\pi a b g\left(\rho_{s}-\rho\right) \sin \varphi-F_{x} \\
& \left(m+m_{y}\right) \dot{v}_{x}+\left(m+m_{x}\right) v_{x} \dot{\varphi}=\rho \Gamma v_{y}-\pi a b g\left(\rho_{s}-\rho\right) \operatorname{Cos} \varphi-F_{y} \\
& \left(I+I_{a}\right) \ddot{\varphi}=\left(m_{x}-m_{y}\right) v_{x} v_{y}-\kappa
\end{aligned}
$$


where $\mathrm{a}$ and $\mathrm{b}$ are half of major and minor axis of ellipse, respectively. Also, $\Gamma(V, \dot{\varphi})$ is the total circulation of the system (defined as equation 5 ), $\rho_{\mathrm{s}}$ is solid density and $m_{\mathrm{x}}, \mathrm{m}_{\mathrm{y}}$, I and $\mathrm{I}_{\mathrm{a}}$ are added mass in $\mathrm{x}$ and $\mathrm{y}$ direction, moment of inertia and added moment of inertia, respectively. Moreover, $\mathrm{F}_{\mathrm{x}}\left(v_{x}\right)$ and $\mathrm{F}_{\mathrm{y}}\left(v_{y}\right)$ and $\kappa(\dot{\varphi})$ are lift, drag and dissipative torque, respectively [19]. The values of $F_{x}, F_{y}$ and $\kappa$ are determined by equation 7 [19].

$\left(\begin{array}{l}F_{x} \\ F_{y}\end{array}\right)=\rho C(A-B \cos 2 \varphi) \sqrt{v_{x}^{2}+v_{y}^{2}}\left(\begin{array}{l}v_{x} \\ v_{y}\end{array}\right)$

$\kappa=\pi \rho C^{4}\left(\frac{V}{C} \mu_{1}+\mu_{2}|\dot{\varphi}|\right) \dot{\varphi}$

As can be seen from equations 5 and 7, this model has 6 tuning parameters as $C_{T}, C_{R}, A$, $\mathrm{B}, \mu_{1}$ and $\mu_{2}$ which cause this model becomes more complicated than Belmonte model. The numerical values for aforementioned tuning parameters are indicated by Andersen et al [19] as:

$C_{T}=1.2, \quad C_{R}=\pi, \quad A=1.4, \quad B=1$

while $\mu_{1}$ and $\mu_{2}$ have been supposed to be varied. Based on Andersen model, a 3DOF model for a thin flat plate in the resisting medium is as follow:

$\tilde{I} \dot{v}_{x}-(\tilde{I}+1) v_{y} \dot{\varphi}=-\frac{v_{y}}{\pi C^{2}}\left(C_{T} V C \sin 2 \varphi+2 C_{R} C^{2} \dot{\varphi}\right)-\frac{V v_{x}}{\pi C}(A-B \cos 2 \varphi)$

$(\tilde{I}+1) \dot{v}_{y}+\tilde{I} v_{x} \dot{\varphi}=\frac{v_{x}}{\pi C^{2}}\left(C_{T} V C \sin 2 \varphi+2 C_{R} C^{2} \dot{\varphi}\right)-\frac{V v_{y}}{\pi}(A-B \cos 2 \varphi)$

$\frac{C^{2}}{4}\left(\tilde{I}+\frac{1}{2}\right) \ddot{\varphi}=-v_{x} v_{y}-C^{2}\left(\frac{\widetilde{U}}{C} \mu_{1}+\mu_{2}|\dot{\varphi}|\right) \dot{\varphi}$

where $\tilde{I}$ is non-dimensional moment of inertia which is defined as $\tilde{I}=\frac{\rho_{s} d}{\rho_{f} c}[19]$ where $\rho_{\mathrm{s}}$ and $\rho_{\mathrm{f}}$ are density of the plate and the fluid, respectively. Also, $\mathrm{d}$ and $\mathrm{C}$ represent the thickness and the chord of the plate, respectively. Moreover, in equation 9, parameter $\widetilde{U}$ represents the descending velocity of the falling object through the fluid and is defined as $\widetilde{U}=\sqrt{g d\left(\frac{\rho_{s}}{\rho_{f}}-1\right)}$ which $\mathrm{g}$ is the gravity. Despite they stated that a transition from fluttering to tumbling will occur with increasing $\tilde{I}$ which is in agreement with the report of Wilmarth [26] and Belmonte et al [20], but the figures in Andersen et al paper show a returning transition (i.e. transition from fluttering to tumbling and return to fluttering) with increasing $\tilde{I}$. In other words, there is two amounts of $\tilde{I}$ correspond to the transition that is in contrast with the finding of Wilmarth [26] and Belmonte et al [20] who pointed out the only one transient value. Anderson et al [19] indicated that $\tilde{I}=1.219$ for transition from fluttering to tumbling, whereas in higher amount of $\tilde{I}(\tilde{I}=3.0)$, the fluttering mode occurred again and for the values of $\tilde{I}$ between these transient values, tumbling mode will happen.

\subsection{Equation of rotation of an articulated plate in the fluid flow}


This section elaborates the development of the equation of rotation of an articulated plate (1 DOF problem) through the fluid current from the aforementioned 3DOF models (i.e. equations 3 and 9).

To obtain the equation of $1 \mathrm{DOF}$ problem (see Figure 1), at first, the velocity components are found at the center of pressure of the plate as:

$v_{x}=V \sin \varphi, \quad v_{y}=-V \cos \varphi-f C \dot{\varphi}$

where $f$ is center of pressure that is determined from mid-chord of the plate. Based on literatures, the $f$ can be calculated analytically for the flat plate. One of the well-known attempts to find an analytical expression for this parameter is known as Kirchhoff-Rayleigh theory [27]. Using the conformal mapping technique in this theory, the center of pressure of the plate in Figure 1 is expressed as:

$X_{c p}=f=\frac{0.75 \operatorname{Cos}\left(\varphi-\frac{\pi}{2}\right)}{4+\left|\pi \operatorname{Sin}\left(\varphi-\frac{\pi}{2}\right)\right|}$

On the other hand, the acceleration components (equation 12) are derived by doing a time derivation on the velocity components.

$\dot{v}_{x}=V \dot{\varphi} \cos \varphi, \quad \dot{v}_{y}=V \dot{\varphi} \sin \varphi-\dot{f} C \dot{\varphi}-f C \ddot{\varphi}$

The dynamical equation of $1 \mathrm{DOF}$ rotating plate is easily found by collecting the torques on the plate, as explained in appendix A. Such an equation which is found by Belmonte model, hereinafter called "RFB model", is as:

$\ddot{\varphi}+\left(2-\frac{12 \pi}{F r^{2}}\right) f \sin \varphi \frac{V}{C} \dot{\varphi}$

$-\left[f \delta_{\perp} \cos \varphi+\left(6 \pi-2 \pi f \operatorname{sgn}\left(v_{x}\right)\right) \sin 2 \varphi\right] \frac{V^{2}}{F r^{2} C^{2}}+\frac{12 \delta_{\omega}}{F r^{2}}|\dot{\varphi}| \dot{\varphi}=0$

Another 1DOF equation is made by Andersen model (henceforth referred to "RFA model") as follow:

$$
\begin{aligned}
(0.25 \tilde{I}+0.125) \ddot{\varphi}+ & {\left[2\left(\tilde{I}-\frac{C_{R}}{\pi}\right) f \frac{V}{C} \sin \varphi+\frac{\widetilde{U}}{C} \mu_{1}\right] \dot{\varphi} } \\
& -\left[\frac{f}{\pi}(A-B \cos 2 \varphi) \cos \varphi+\left(0.5+\frac{f C_{T}}{\pi} \sin \varphi\right) \sin 2 \varphi\right] \frac{V^{2}}{C^{2}}+\mu_{2}|\dot{\varphi}| \dot{\varphi}=0
\end{aligned}
$$

As can be observed, equation 14 (RFA model) is more complicated than equation 13 (RFB model). As said before, the added moment of inertia has not been considered in Belmonte model. In an overview, RFA model consists of more terms in each part (i.e. inertia, damping and exciting parts) in comparison to RFB model. Although the existing of more terms in each part of the RFA make it an intricate model, but in positive point of view, the RFA model is capable to be tuned up appropriately to obtain more accurate results. 


\subsection{Non-dimensional Form and Homogenization of Equations}

Both of the RFA and RFB (Equation 12 and 14) are written in dimensionless form using the time scale, $\tau$, and the velocity scale, $\mathrm{U}$, which are defined by the expressions:

$$
\tau=\frac{C}{V} \quad, \quad U=\Omega C
$$

where $\mathrm{C}$ is the plate chord and $\mathrm{V}$ is the current velocity. The following non-dimensional parameters are constructed from the characteristics scales (equation 15).

$$
t^{*}=\frac{t V}{C}, \quad \dot{\tilde{\varphi}}=\frac{\dot{\varphi} C}{V}, \quad \tilde{\varphi}=\varphi
$$

Using the parameters in equations 15 and 16, the non-dimensional form of RFB model is obtained as follow:

$$
\begin{aligned}
F r^{2} \frac{d \dot{\tilde{\varphi}}}{d t^{*}}+ & \left(2 F r^{2}-12 \pi\right) f \sin \tilde{\varphi} \dot{\tilde{\varphi}} \\
& -\left[f \delta_{\perp} \cos \tilde{\varphi}+(6 \pi-2 \pi f \operatorname{sgn}(\sin \tilde{\varphi})) \sin 2 \tilde{\varphi}\right]+12 \delta_{\omega}|\dot{\tilde{\varphi}}| \dot{\tilde{\varphi}}=0
\end{aligned}
$$

And, the dimensionless form of RFA model is expressed as:

$$
\begin{aligned}
(0.25 \tilde{I} & +0.125) \frac{d \dot{\tilde{\varphi}}}{d t^{*}}+\left[2\left(\tilde{I}-\frac{C_{R}}{\pi}\right) f \sin \tilde{\varphi}+\frac{\widetilde{U}}{V} \mu_{1}\right] \dot{\tilde{\varphi}} \\
& -\left[\frac{f}{\pi}(A-B \cos 2 \tilde{\varphi}) \cos \tilde{\varphi}+\left(0.5-\frac{2 f C_{T}}{\pi} \sin \tilde{\varphi}\right) \sin 2 \tilde{\varphi}\right]+\mu_{2}|\dot{\tilde{\varphi}}| \dot{\tilde{\varphi}}=0
\end{aligned}
$$

In equations 17 and 18, Fr and $\tilde{I}$ represent the same concept of mass moment of inertia so that they can be synchronized by definition of a new dimensionless moment of inertia ( $\left.I^{*}\right)$, using the moment of inertia scale, $\varepsilon=\rho C^{5}[28,29]$, as $\mathrm{I}^{*}=\mathrm{I} / \rho \mathrm{C}^{5}$. The relation between these dimensionless parameters (i.e. Fr, $\tilde{\mathrm{I}}$, and $\mathrm{I}^{*}$ ) is as below:

$$
F r=\sqrt{12 I^{*}} \text { and } \quad \tilde{I}=12 I^{*}
$$

To obtain good results in simulation of fluttering and autorotation of an articulated plate, the unknown coefficients need to be determined as accurate as possible. For determining of unknown coefficients, the iterating method has been selected to match the result of equations 17 and 18 with experimental data. To execute such a method, the aforementioned equations are solved numerically for a given $I^{*}$ while the values of unknown coefficients alter repetitively till the best match is obtained. The procedure of finding the unknown coefficient is elaborated as: for each set of value of $I^{*}$ and coefficients, the error of dynamical characteristics (i.e. angle of rotation and angular velocity) is evaluated by comparing the numerical results to the experimental data and this cycle is repeated while the new values have been set for coefficients and I* is kept the same. This method applied to several $\mathrm{I}^{*} \mathrm{~s}$ (with wide range from low to very high) and eventually, the best values of unknown coefficients have been chosen for RFB model as: 
For RFA model, the following values are proposed:

$$
\begin{aligned}
A=45.1 I^{* 2}, \quad B=37.58 I^{* 2}, \quad C_{T}=52.61 I^{* 2}, \\
C_{R}=13.25 \pi I^{*}, \quad \mu_{1}=0, \quad \text { and } \mu_{2}=0.045 \pm 0.035
\end{aligned}
$$

As can be seen, $\mu_{2}$ changes in wide range to reach the good result of fluttering and autorotation. The value of $\mu_{2}$ is increased by increasing $I^{*}$ and vice versa.

\section{Experimental Setup}

In order to measure the dynamics of rotation of an articulated plate in the current, some experiments have been carried out in laboratory of wave and current (LOC) at Federal University of Rio de Janeiro (UFRJ).

\subsection{Model Setup}

As mentioned in foregoing paragraphs, the autorotation will occur with large enough moment of inertia. In the water channel, increase the dimensionless moment of inertia is not as easy as wind tunnel in virtue of higher density of water in comparison with air [30]. For this purpose a unique apparatus (see Figure 2) has been designed to change the moment of inertia of the plate in water flume. In this apparatus, a bar is installed on top of the plate. The two $4 \mathrm{Kg}$ masses are added to the system, which could be placed in different positions along the bar to provide the required moment of inertia. The plate is supported by two ball bearings at top and bottom of axis of rotation. Resistance of bearings against rotation comes from friction between balls and cast.

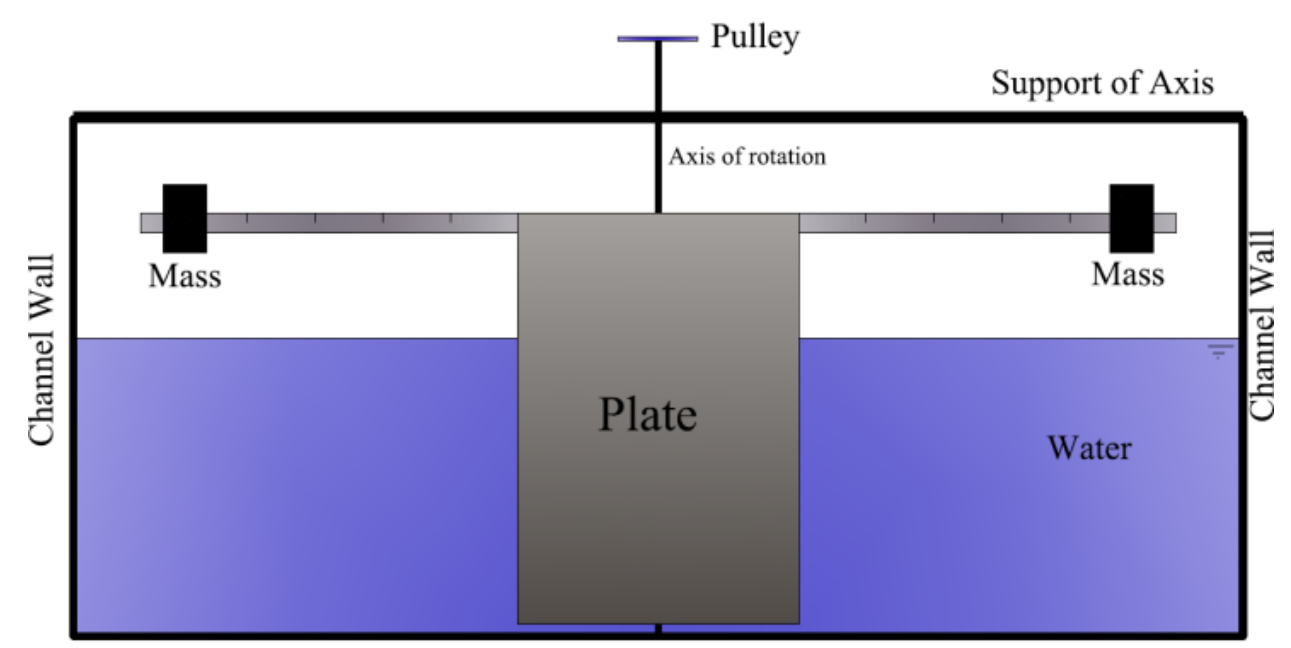

Figure 2: The experimental setup for providing different mass moment of inertia on the plate in front view.

The water depth was set to $0.5 \mathrm{~m}$. Uniform main flow is produced by four centrifugal water pumps. The maximum achievable current velocity in the channel is $0.5 \mathrm{~m} / \mathrm{s}$ which corresponds to Hydraulic Froude number $\left(F r_{h}=V / \sqrt{g H}\right)$ of 0.22 . According to Fr the flow state is subcritical or tranquil flow. 
The uncertainty ${ }^{1}$ of the current velocity in this paper is less than 1 percent. The fluid properties are shown in Table 1.

Table 1: The fluid properties

\begin{tabular}{rc}
\hline \multicolumn{2}{c}{ Fluid properties } \\
\hline Fluid type & Fresh water \\
Density & $1000 \mathrm{~kg} / \mathrm{m}^{3}$ \\
Velocity range & $0.07-0.335 \mathrm{~m} / \mathrm{s}$ \\
Flow Reynolds & $21000-100000$ \\
Number & \\
Temperature & $20 \mathrm{deg}$ \\
Current depth & $0.5 \mathrm{~m}$ \\
\hline
\end{tabular}

Important characteristics of the plate are listed in Table 2.

Table 2: Characteristics of the experimented model.

\begin{tabular}{ll}
\hline \multicolumn{2}{c}{ Blade properties } \\
\hline $\begin{array}{l}\text { Width projected on perpendicular of } \\
\text { current direction }\end{array}$ & $0.3 \mathrm{~m}$ \\
Materials & Aluminum \\
Thickness (d) & $5 \mathrm{~mm}$ \\
Height & $0.6 \mathrm{~m}$ \\
\hline
\end{tabular}

Using the dimensional analysis, the following non-dimension numbers are found.

Table 3: Non-dimensional parameters which affect the rotation of plate in the water

\begin{tabular}{|c|c|c|c|c|}
\hline $\begin{array}{c}\text { Angle of } \\
\text { Rotation }\end{array}$ & $\begin{array}{c}\text { Reynolds } \\
\text { number }\end{array}$ & $\begin{array}{c}\text { Thickness } \\
\text { ratio }\end{array}$ & $\begin{array}{c}\text { Dimensionless mass } \\
\text { moment of inertia }\end{array}$ & $\begin{array}{c}\text { Aspect } \\
\text { ratio }\end{array}$ \\
\hline$\theta_{r}$ & $\mathrm{Re}=\mathrm{VC} / v$ & $\tau=\mathrm{d} / \mathrm{C}$ & $I^{*}=I_{s} / \rho_{w} C^{5}$ & $\mathrm{Ar}=\mathrm{H} / \mathrm{C}$ \\
\hline
\end{tabular}

The dimensionless mass moment of inertia $\left(I^{*}\right)$ is defined as the ratio of the structural mass moment of inertia to the hydrodynamic added moment of inertia [31]. In this study, $\tau$ and Ar are constant and equal to 0.0166 and 1.66 , respectively.

\subsection{Dynamic Measurement of Fluttering and Autorotation}

In order to measure dynamical characteristics of an articulated flat plate such as angular velocity, angular acceleration and also angular position with respect to time, a pack of wireless sensors was set up. This pack included a microcontroller, some sensors included accelerometer, a rategyro and a magnetometer and two wireless communication modules.

Arduino Uno is used as microcontroller device in this study. The Arduino is an opensource computer that designs and manufactures microcontroller-based kits for building digital devices and interactive objects that can sense and control objects. Moreover, the wireless

\footnotetext{
${ }^{1}$ Uncertainty applies to predictions of future events, to physical measurements that are already made. It is the standard deviation ( $\sigma$ ) divided by the square root of the number of measurements (n).
} 
communication is undertaken by two Xbee-pro 802.15.4 series 1 (S1) modules. The XBee radio modules were configured to communicate with 19200 as its Baud rate.

As mentioned in foregoing paragraph, dynamic characteristics of the rotating articulated plate have been measured by sensors included accelerometer, rategyro for measuring angular velocity and magnetometer for measuring angular position. In this study, one sensor is used which gathered together all three sensors into one. UM7-LT (as shown in Figure 3) is a 3rdgeneration Attitude and Heading Reference System (AHRS) that takes advantage of state-ofthe-art MicroElectroMechanical Systems (MEMS) technology to improve performance and reduce costs. The UM7 communicates using a 3.3V TTL UART ${ }^{2}$ at user-configurable baud rates ranging from 9600 baud to 921600 baud. In this study, binary packet is utilized for communication. The data transmission in binary method is carried out using complicated commands which should be sent to sensor via microcontroller. Based on receiving commands by sensor, the sensor send data to microcontroller. All binary packets sent and received by the UM7 should conform to a special format which has been elaborated in sensor datasheet. The transmission rate used in this study has been arranged for $100 \mathrm{~Hz}$.

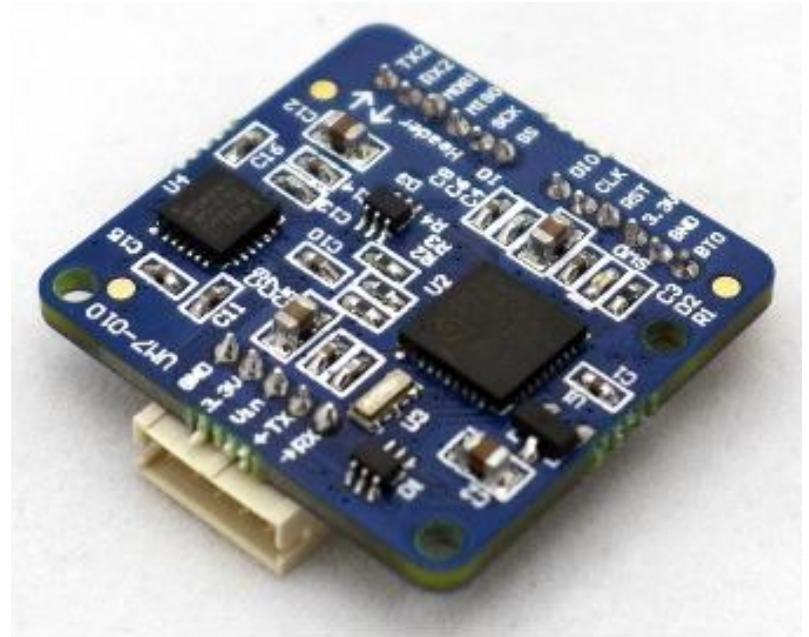

Figure 3: UM7 sensor including Accelerometer, Rate gyro and magnetometer.

\subsection{Installation the sensor on the Model}

The pack of sensor is installed on the model of an articulated plate as shown on the bottom of Figure 4. This pack is supplied by 9V lithium-Ion rechargeable battery in which is mounted next to the microcontroller, as shown on the top of Figure 4. The package put on the axis of rotation to diminish its effect on moment of inertia.

\footnotetext{
${ }^{2}$ UART (Universally Asynchronous Receiver/Transmitter) is used to receive and transmit data serially. UART transmits one bit at a time at a specified data rate which sometimes, this method of serial communication is referred to TTL (TransistorTransistor Logic).
} 

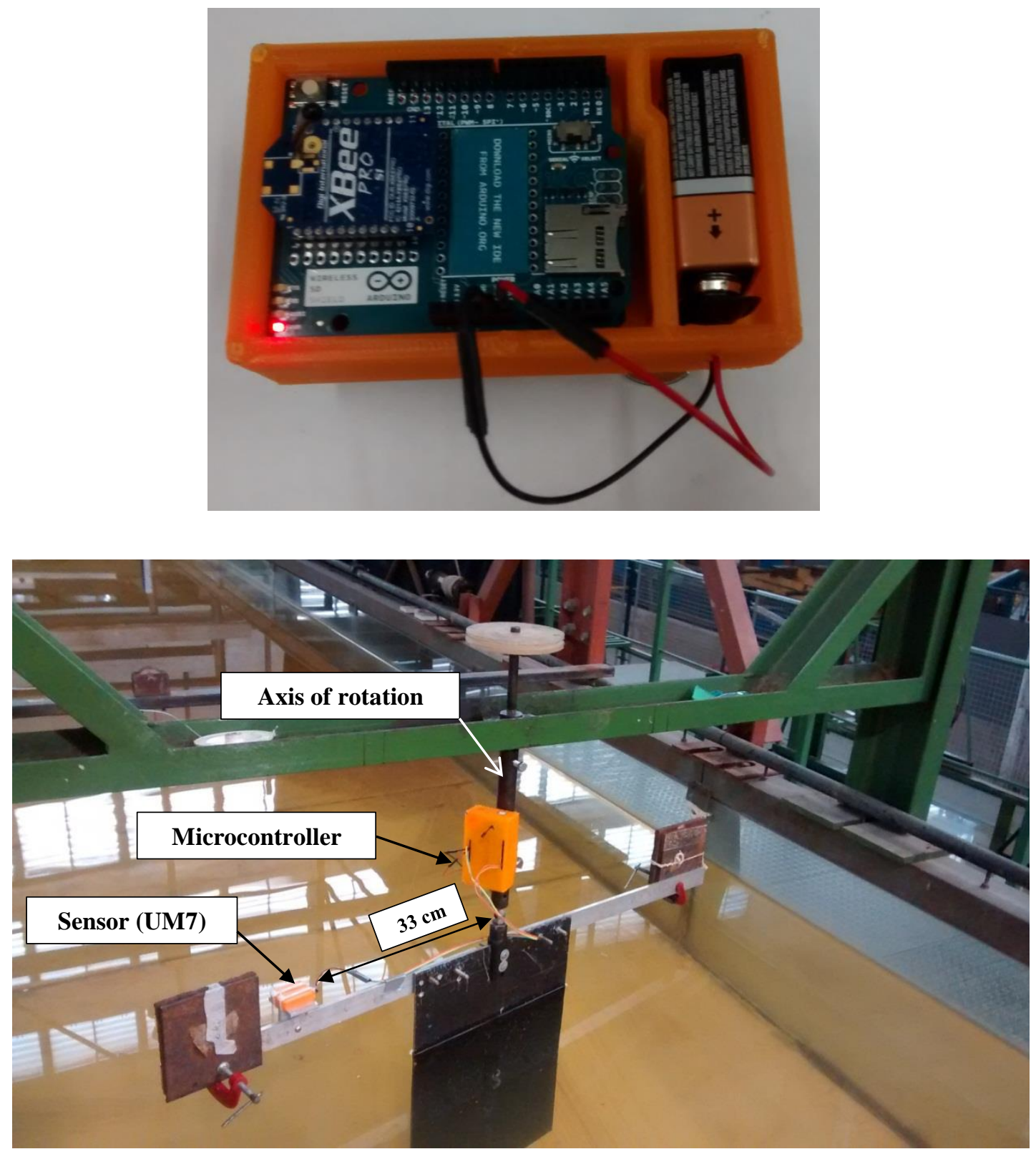

Figure 4: Top: package of battery and microcontroller. Bottom: set up of the sensor (MU7) and the microcontroller on the model.

\section{Result and Discussion}

In the following section, the results of both models are shown with experimental data. Values of the important parameters are given in captions of each figure. In addition, nonlinear analysis is performed on RFA model and bifurcation diagram is presented for this model.

\subsection{Dynamical Characteristics}

Figure 5 shows the comparison of results of the RFA and the RFB models to experimental data. This figure, considered the result of angle of rotation (left column) and angular velocity (right column) for the wide range of $\mathrm{I}^{*}$. In matrix of Figure 5, row A shows the comparison of fluttering mode, row $\mathrm{B}$ corresponds to chaotic mode (mixing of Autorotation and fluttering) and also, rows $\mathrm{C}$ to $\mathrm{F}$ are dedicated to the result of autorotation mode. 

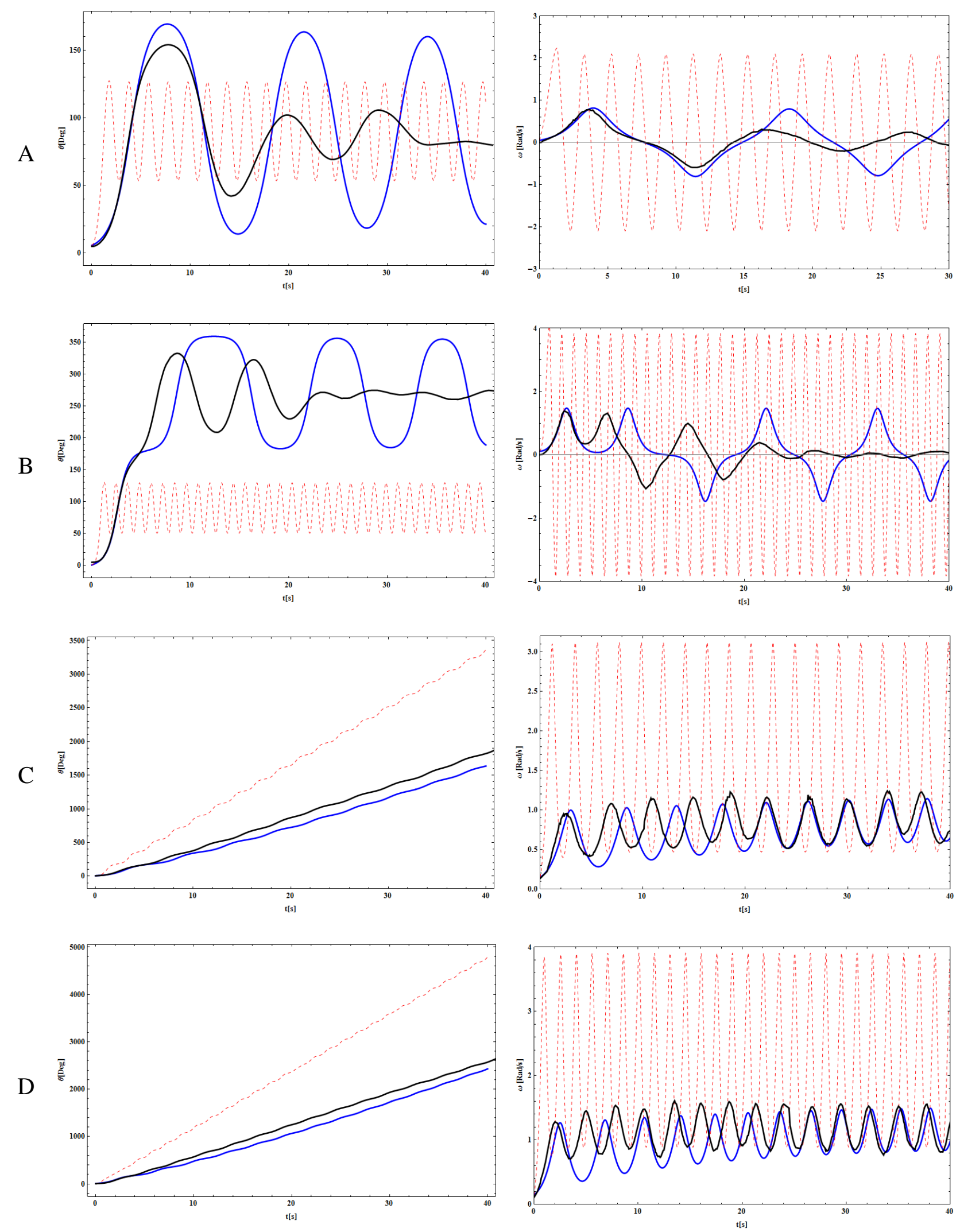

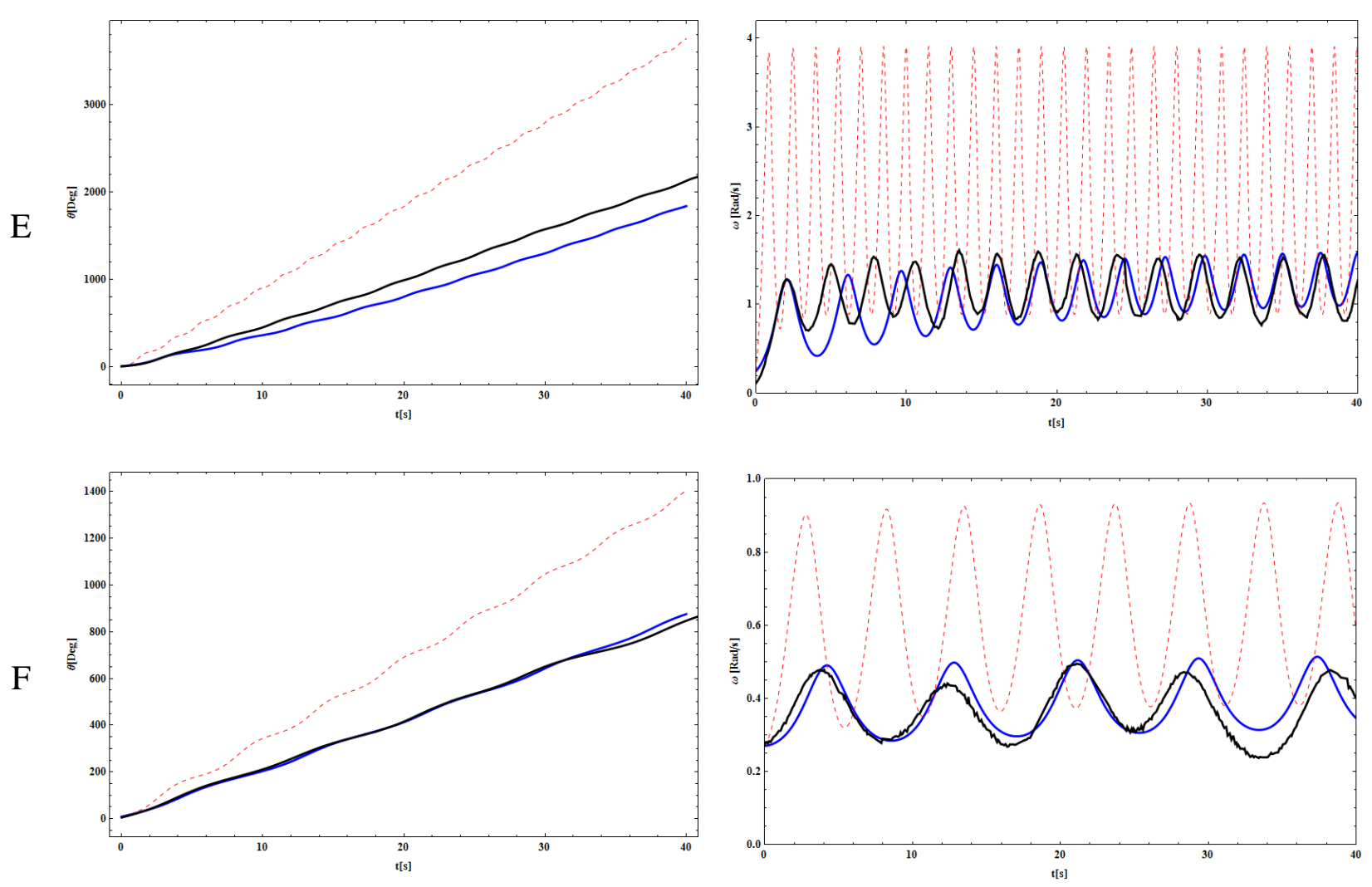

Figure 5: Angle of rotation (left column) and angular velocity (right column) for I*, Res and $\mu_{2}$ of A: 0.044 $\& 37000 \& 0.0095, \mathbf{B}: 0.054 \& 70000 \& 0.01, \mathbf{C}: 0.212 \& 70000 \& 0.035, \mathbf{D}: 0.249 \& 92000 \& 0.041, \mathbf{E}$ : $0.315 \& 70000 \& 0.052$, and F: $0.566 \& 32000 \& 0.081$. Note that dashed red line, solid thick blue line and solid thick black line represent the result of RFB model, RFA model and experiment, respectively.

Table 4 lists the frequency of rotation $(f)$ and statistical data included the average $(\overline{\dot{\varphi}})$ and the standard deviation $(\delta \dot{\varphi})$ which have been obtained by the analysis of angular velocity of the former figures. By comparing the frequency of rotation between experimental data and RFA and RFB models, it is concluded that the frequency of RFA model is close to the frequency of experiment whilst the frequency of RFB model is at least double. Accordingly, based on the figures, the plate which is simulated by RFB model rotates or oscillates much faster than either the simulated plate by FRA or experimented plate.

Table 4: Statistical data and frequency of rotation associated with data of experiment and also RFA and RFB.

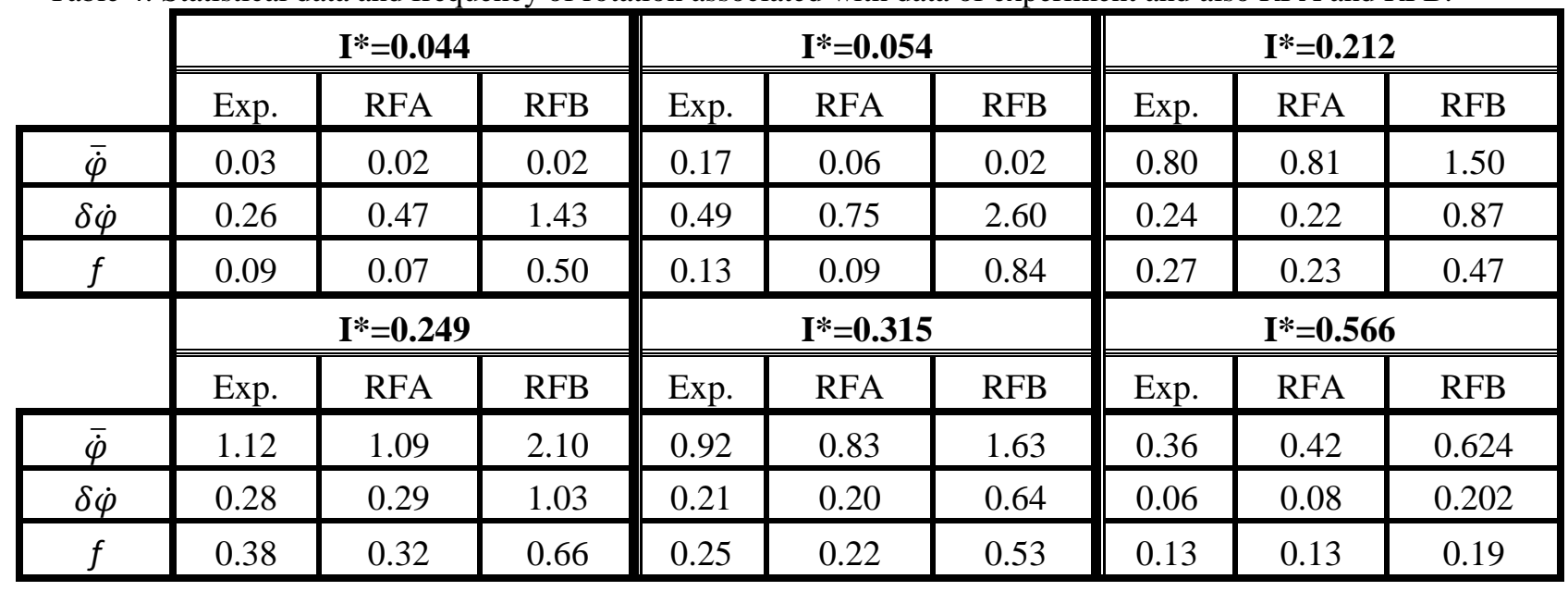


The statistical data demonstrate that the results of RFA model and experiment are in the same order whereas the results of RFB model show a significant discrepancy with the experiment. Broadly speaking, according to the figures and also Table 4, the RFA model is more appropriate to simulate the rotation modes of an articulated plate through the fluid flow.

Furthermore, it can be stipulated that the simulation of fluttering mode by analytical approaches is more complicated in comparison to autorotation mode. Such a difficulty occurs due to complexity of vortex field in fluttering mode. Suppose a $2 \mathrm{D}$ plate that is rotating in the fluid flow and also two vortices that are forming on each edge of the plate with contra rotating against each other. According to laboratory observations, the formation and developing of the vortices will be disturbed by oscillation rotation of the plate. In other words, oscillation motion of the plate in fluttering mode interrupts formation of the vortex on the edges of the plate. Consequently, the oscillation creates a complex field of vorticity around the plate so that it is difficult to assign an analytical formula for total circulation of fluttering mode, as easily as defined by Belmonte et al and Andersen et al. On the other side, analysis of vorticity around the rotating object is fairly straightforward. Therefore, it is possible to allot the fairly accurate formula to the total circulation around the continuous rotating plate.

\subsection{Phase Diagram}

\subsubsection{Rotation Angle- Angular Velocity}

Phase diagrams of angle of rotation vs angular velocity have been depicted in Figure 6 for various $I^{*}$ s. Among these figures, figure $\mathrm{A}$ and $\mathrm{B}$ represent phase diagrams of fluttering and chaotic motion, respectively and the others represent the phase diagram of autorotation mode. These pictures also approve that the RFA simulates the angle and velocity of motion of all modes (fluttering, chaotic and autorotation) more accurate than the FRB. The first oscillation in fluttering mode is properly modeled by the RFA. In further oscillation, fluttering decays rapid in experiment while the simulated motion by the RFA decays much more slowly. Despite of low accuracy in the modeling of the decaying of oscillation motion, the RFA model simulates the autorotation with good accuracy.

As shown in picture A of Figure 6, the amplitude of oscillation predicted by RFB is half of the results from the FRA whereas the amplitude of velocity is double of the RFA. In other words, fluttering mode simulated by RFB progresses faster with lower amplitude. Regarding to pictures $\mathrm{C}$ to $\mathrm{F}$ of Figure 6 , the amplitude of angular velocity and angle of rotation in autorotation mode calculated by the RFB, is at least double of the result from the RFA for different $I^{*}$. 

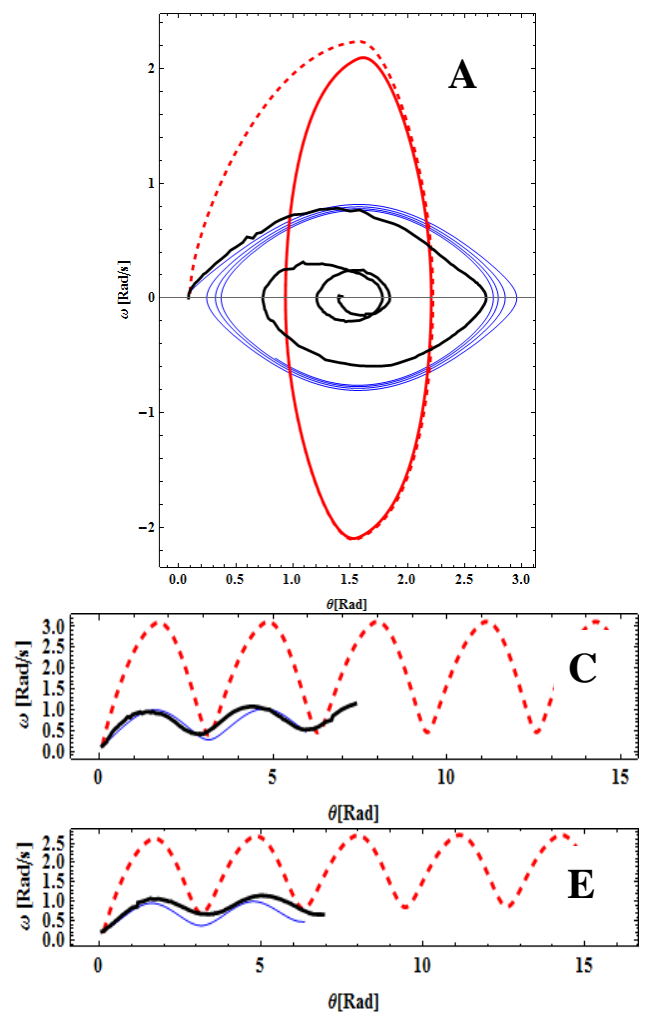
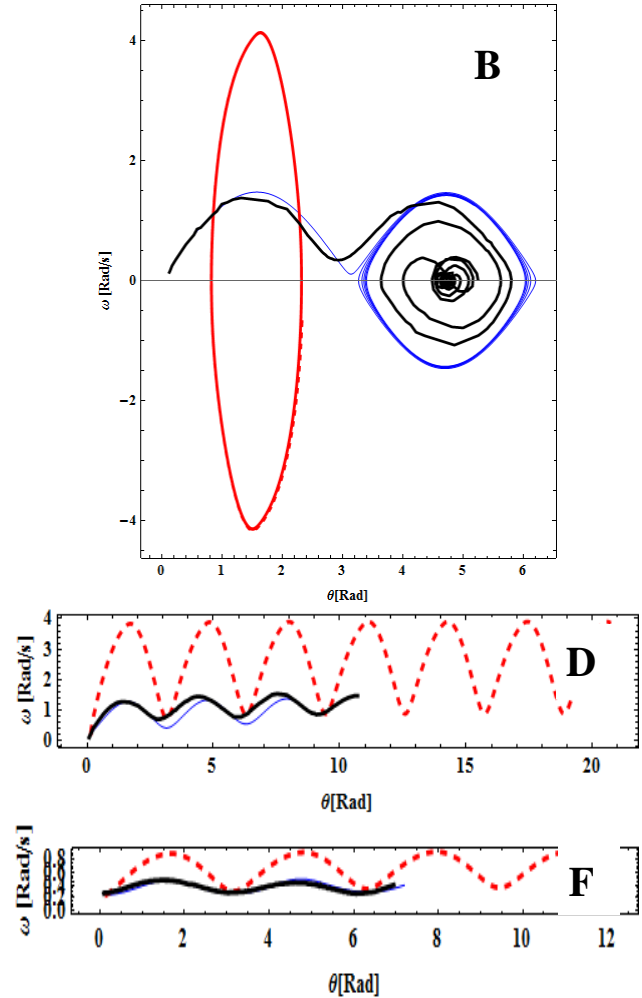

Figure 6: Phase diagram of Angle of rotation and angular velocity for I*, Res and $\mu_{2}$ of A: $0.044 \& 37000$ $\& 0.0095$, B: $0.054 \& 70000 \& 0.011$, C: $0.212 \& 70000 \& 0.035$, D: $0.249 \& 92000 \& 0.041$, E: $0.315 \&$ $70000 \& 0.052$, and F: $0.566 \& 32000 \& 0.081$. Note that dashed red line, solid thin blue line and solid thick black line represent the result of RFB model, RFA model and experiment, respectively.

\subsubsection{Angular Velocity-Angular Acceleration}

Based on pictures of Figure 7, the RFB is over-calculating the dynamical characteristics of the motion whereas the RFA calculates them very well. According to the pictures, the angular acceleration calculated by the RFB is at least 6 times greater than experimental data whereas the result of RFA is almost equal to the experiment. As can be expected, by increase in I*, angular acceleration and angular velocity decrease. However, low value of $I^{*}$ gives the similar result, as well. In other words, there is an optimum value for $I^{*}$ to reach the maximum value of angular velocity and acceleration.

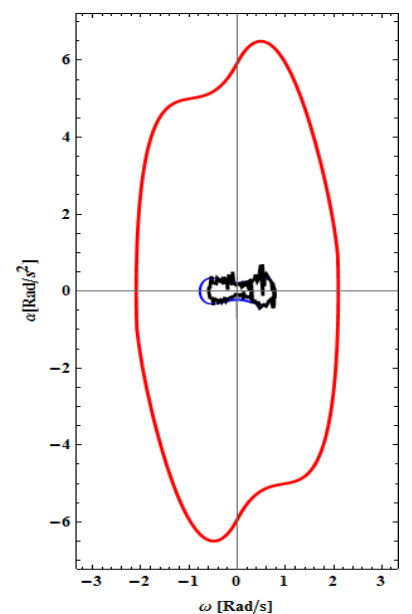

$\mathbf{A}$

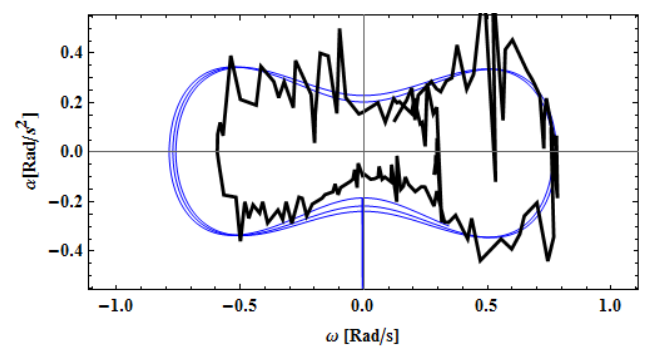



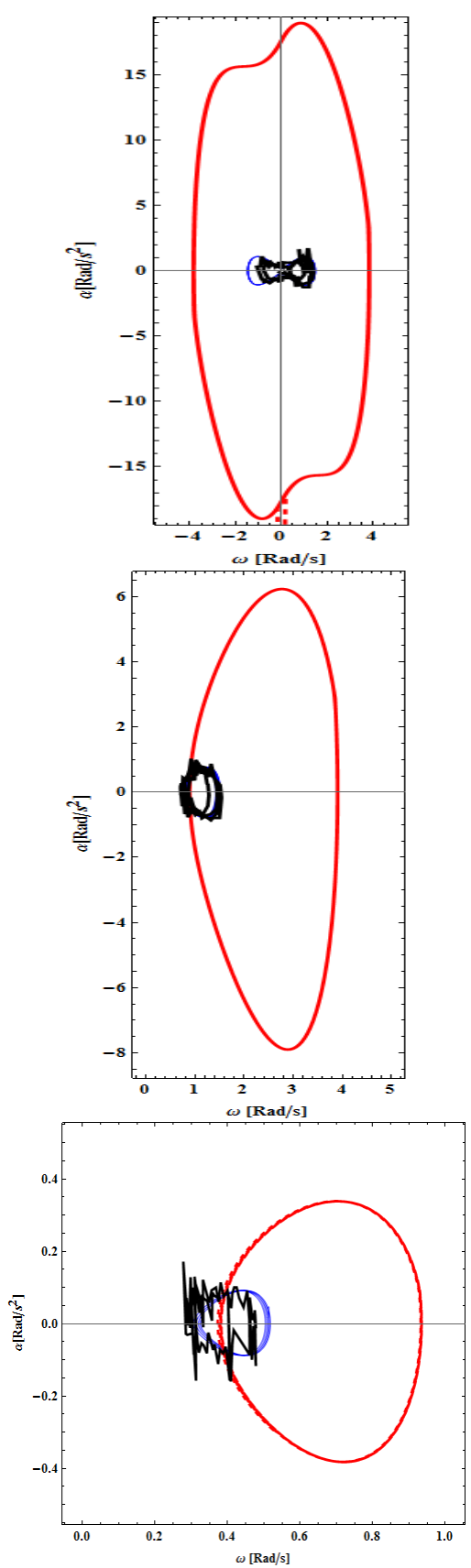

B

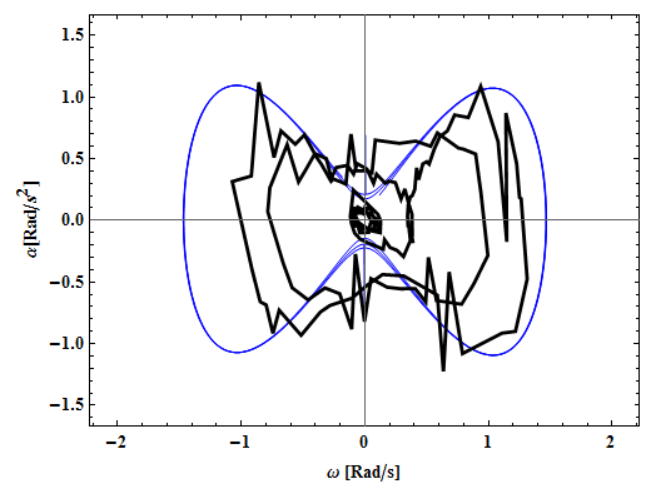

$\mathbf{C}$
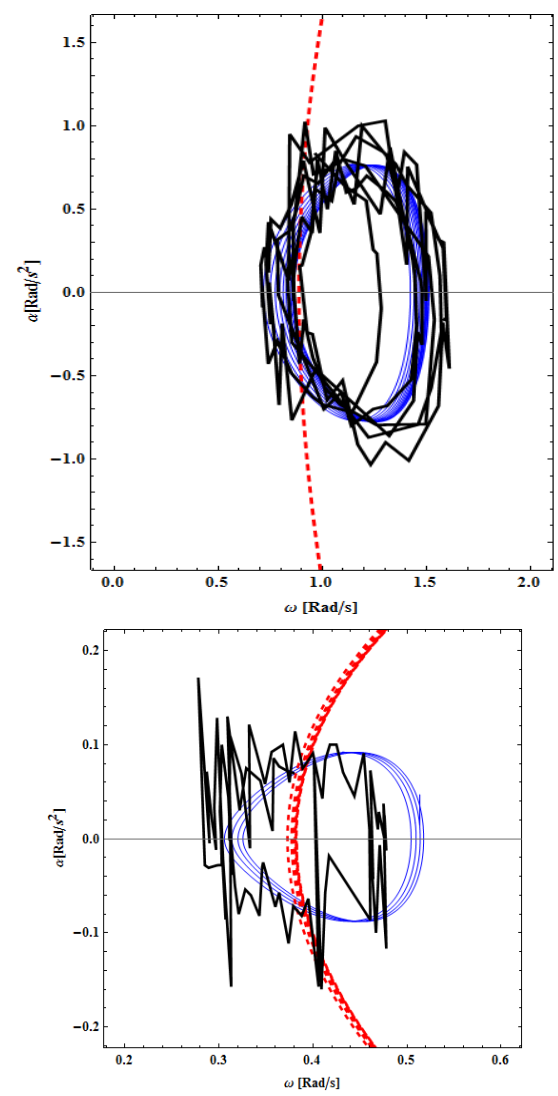

Figure 7: Phase diagram of angular acceleration vs angular velocity for I* and Res of A: $0.044 \& 37000 \&$ 0.0095 , B: $0.054 \& 70000 \& 0.011$, C: $0.249 \& 92000 \& 0.035$, and D: $0.566 \& 32000 \& 0.081$. Note that dashed red line, solid thick blue line, and solid thick black line represent the results of the model of RFB, RFA, and experiment, respectively.

\subsection{Stability Analysis}

Stability analysis is an important study on nonlinear ordinary differential equations like equation 18. The stability analysis is conducted on the RFA model. For proper analysis, the fixed points of the equation are found at the first step. Equation 18 has general form as

$\dot{\lambda}=q+\left(p \lambda-k \lambda^{2}\right)$

where $\lambda=\dot{\tilde{\varphi}}$. Also, $\mathrm{k}$ is constant coefficient and $p$ and $q$ are variable coefficients that are defined as below. 
$k=\mu_{2} /\left(3 I^{*}+0.125\right)$

$p=2.5 I^{*} f \sin \tilde{\varphi} /\left(3 I^{*}+0.125\right)$

$q=\frac{f I^{* 2}}{\pi\left(3 I^{*}+0.125\right)}\left[(45.1-37.58 \cos 2 \tilde{\varphi}) \cos \tilde{\varphi}+\left(\frac{0.5 \pi}{f I^{* 2}}-105.2 \sin \tilde{\varphi}\right) \sin 2 \tilde{\varphi}\right]$

In equation 22 , for $\mathrm{q}=0$ the rest terms create a prototypical of transcritical bifurcation whereas if $p=0$ the prototypical of saddle node bifurcation is formed. It is possible to find a threshold for a system using transcritical bifurcation. The fixed points of the parenthesized phrase in equation 22 are $\lambda^{*}=0$ and $\lambda^{*}=p / k$. Intersection of these lines in stability diagram denotes threshold of the present model. Such a threshold is found if $p=0$ that yields:

$P_{t h}=\widetilde{\varphi}_{t h}=n \pi \quad n=0,1,2, \ldots$

For $\mathrm{p}<P_{t h}, \lambda=0$ represents the stable points and $\lambda=p / k$ shows the unstable points whereas, for $\mathrm{p}>P_{t h}$, the line of $\lambda=0$ corresponds to the unstable points and $\lambda=p / k$ demonstrates the stable points. It is worthy to note that for $\mathrm{p}<P_{t h}$, the points on $\lambda=0$ are interpreted physically as a plate into still fluid (the fluid without flow). The aforesaid discussion is depicted in Figure 8.

In Figure 8, the inclined line represents the values of angular velocity in which the body can rotate stably. Thus, in the case of $\mathrm{p}>P_{t h}$, the stable points on $\lambda=\mathrm{p} / k$ represent the autorotation mode and therefore, all the points, in the around, are flown towards such stable points. It can be inferred from the former discussion that the region between horizontal unstable line and inclined stable line (yellow zone in Figure 8) indicates the possibility of the occurring autorotation. This region is dependent on $\mathrm{I}^{*}$ so that an increase in $\mathrm{I}^{*}$ causes to enlarge this area and consequently increases the possibility of the occurring autorotation. 


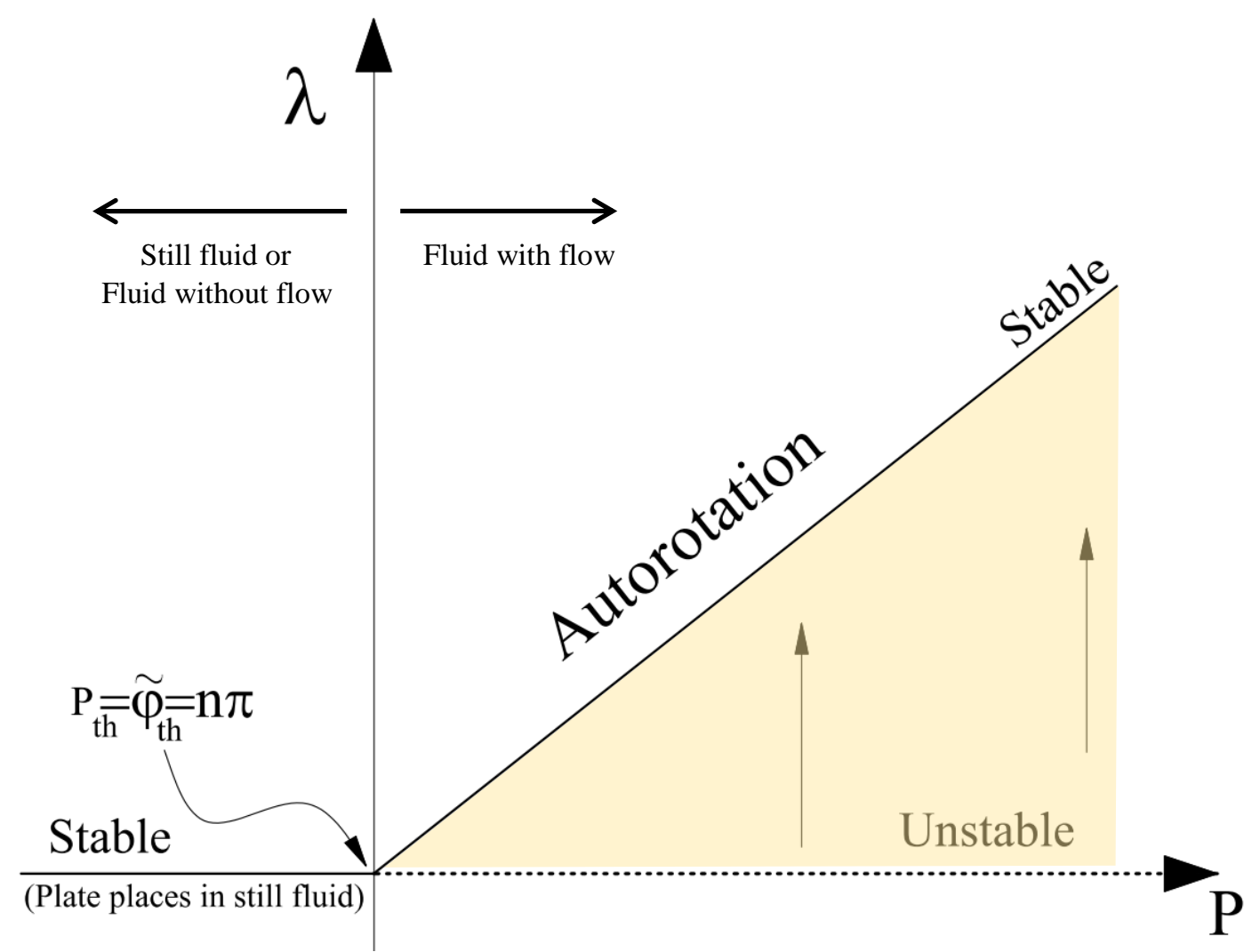

Figure 8: Stability diagram of transcritical bifurcation for equation 22. In this figure, $\lambda=\dot{\tilde{\varphi}}$ and $P=$ $2.5 I^{*} f \sin \tilde{\varphi} /\left(3 I^{*}+0.125\right)$ and $P_{t h}=\tilde{\varphi}_{t h}=n \pi(\mathrm{n}=0,1,2, \ldots)$ is Threshold of the system.

On the other side, if let $\mathrm{p}=0$ and $\lambda=u / k$ in equation 22 , this equation yields:

$\dot{u}=q k-u^{2}$

The last equation represents normal form of the saddle node bifurcation. The fixed points are $u= \pm \sqrt{q k}$ where the real values exist for $\mathrm{qk} \geq 0$. Because of positive value of $\mathrm{k}(\mathrm{k}>0)$, therefore the fixed points are found if $q \geq 0$. Thus:

$\frac{f I^{* 2}}{\pi\left(3 I^{*}+0.125\right)}\left[(45.1-37.58 \cos 2 \tilde{\varphi}) \cos \tilde{\varphi}+\left(\frac{0.5 \pi}{f I^{* 2}}-105.2 \sin \tilde{\varphi}\right) \sin 2 \tilde{\varphi}\right] \geq 0$

Figure 9 shows the periodical roots of $\mathrm{q}$ for different $\mathrm{I}^{*} \mathrm{~s}$. As can be seen, for all $\mathrm{I}^{*} \mathrm{~s}$, there are two regions that $\mathrm{q}$ is positive and saddle node bifurcation might happen. Referring to previous discussion, it can be concluded that $\lambda=\sqrt{q / k}$ is stable point and $\lambda=-\sqrt{q / k}$ is unstable point. The stable point here means that the plate can reach to autorotation mode. Furthermore, Figure 9 demonstrates that in $\varphi=\pi / 2 \mathrm{rad}$, autorotation will happen easier than $\varphi=0$ and $\pi$ however for higher $I^{*}$ such a possibility increases. 


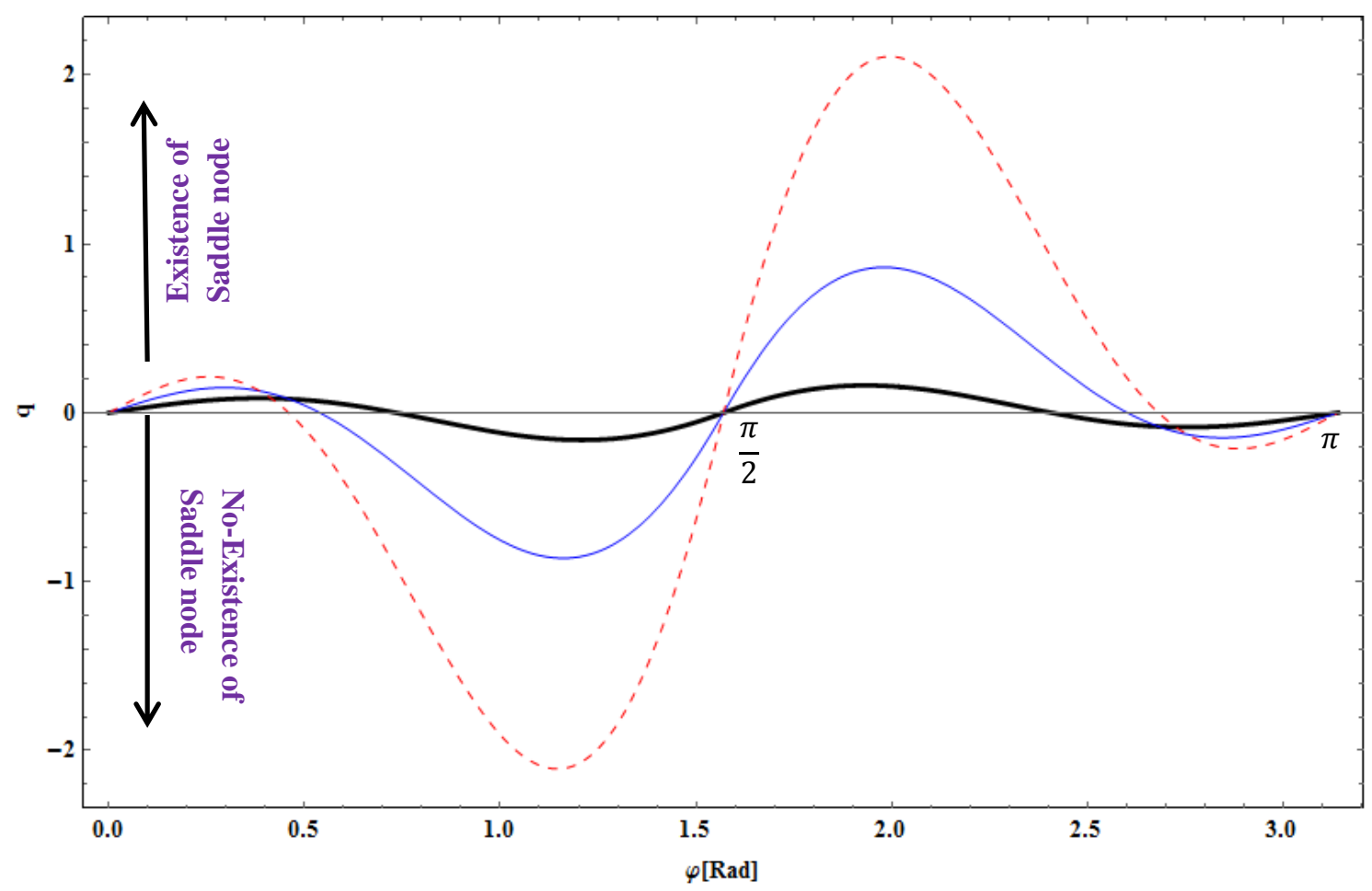

Figure 9: Plot of q (equation 23) versus $\varphi$ for one period in different $I^{*}$. In this plot, black thick line, blue line and red dash line represent $I^{*}=0.5,1.0$, and 1.5 , respectively.

Besides the role of $\mathrm{I}^{*}$ on determination of the rotational mode, initial conditions affect occurring autorotation and fluttering, as well. The initial conditions are combination of initial angle of attack and initial angular velocity. Hence, the phase diagram of the angle of rotation $(\theta)$ versus the angular velocity $(\omega)$ of the RFA model (equation 14) is illustrated in Figure 10 for three different values of a group of the effective parameters (i.e. I*, Re and $\mu_{2}$ ). These figures indicate that, for each combination of $\mathrm{I}^{*}, \mathrm{Re}$, and $\mu_{2}$, there is one bifurcation line (red dashed line in these figures) in which autorotation will happen if the initial conditions are chosen above it. In contrast, for inappropriate initial conditions (i.e. the points below the red line), fluttering will happen. For instance, in Figure $10-\mathrm{A}$, the values of $(\pi / 2,0.14)$ or $(0$ or $\pi$, 0.84) represent the points on the red dashed line, and therefore, autorotation might happen if the angular velocity is chosen greater than the second value of parentheses. On the other side, fluttering will occur if the angular velocity is lower than the mentioned value. It is important to note that, for the initial conditions close to red dashed line, the mode of rotation can change between the autorotation and the fluttering after some cycles. In other words, for initial conditions near the red dashed line, the chaotic rotation might happen. 

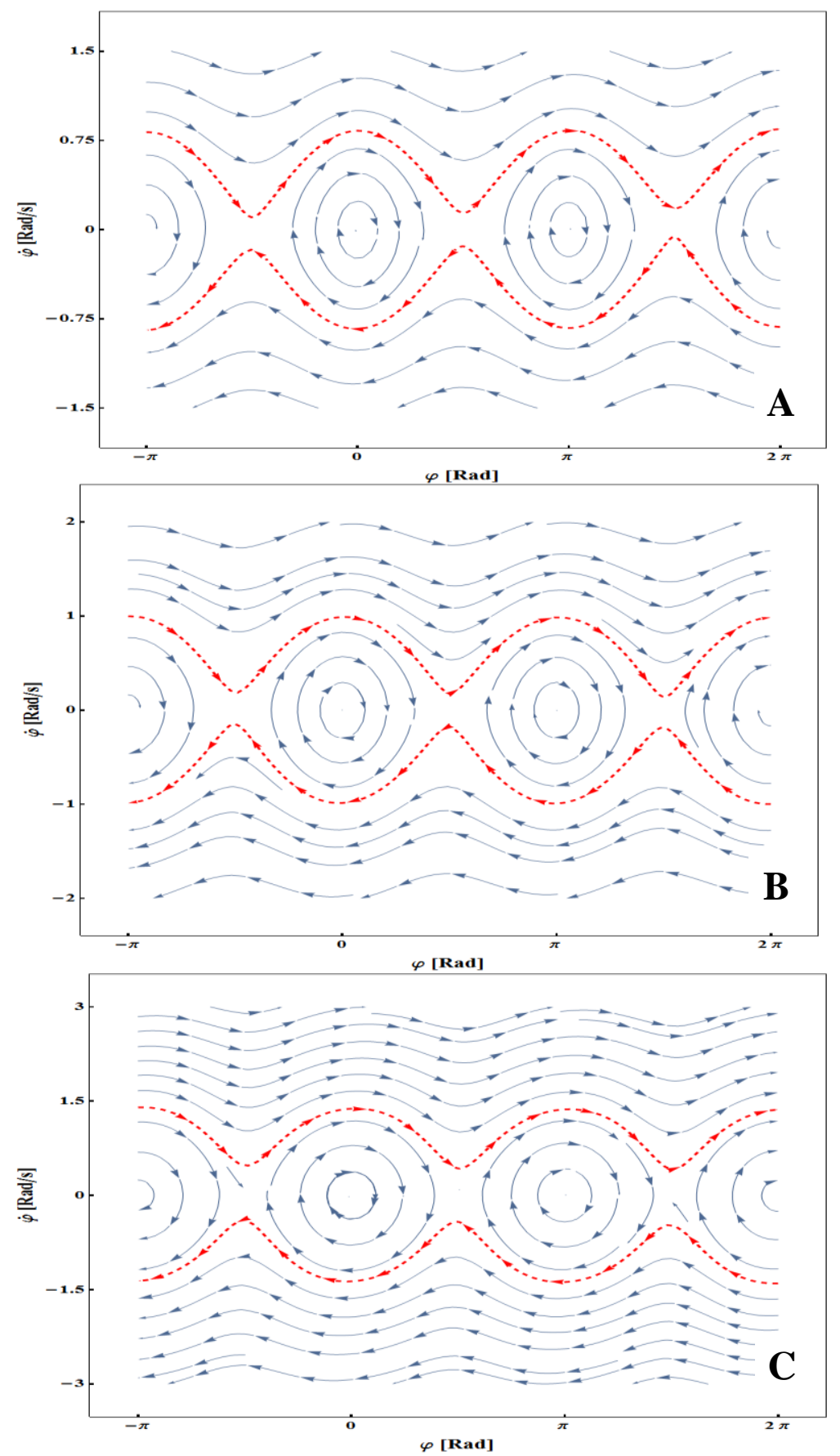

Figure 10: Phase diagram of RFA for A: $I^{*}=0.044, \operatorname{Re}=37000$ and $\mu_{2}=0.006, \mathrm{~B}: \mathrm{I}^{*}=0.212, \mathrm{Re}=70000$ and $\mu_{2}=0.025$, and $\mathrm{C}: \mathrm{I}^{*}=0.391, \operatorname{Re}=100000$ and $\mu_{2}=0.05$. Bifurcation line is indicated by dashed line so that the initial condition below this line brings the plate to fluttering.

The points $\varphi=\pi / 2$ and $\varphi=3 \pi / 2$ correspond to position of the body, with the edge forwards while the fixed points $\varphi=0$ and $\varphi=\pi$ are related to position with the wide side forwards. On the phase portrait, one can see the separatrix (red dashed lines) containing the saddle points. The orbits inside the separatrix correspond to oscillatory motions around the centers, while the rotational motions are represented by curves outside the separatrix.

\subsection{Bifurcation Diagram}

By referring to forgoing sections, the transition from fluttering to autorotation will occur by increasing the mass moment of inertia; however chaotic motion might be observed 
wherein the transition happens. As a result of former section, the RFA model is consider to find the analytical bifurcation diagram of fluttering and autorotation.

Referring to Figure 6, the autorotation will happen if the plate rotates at least one cycle (i.e. $2 \pi$ radian). Thus, the existence of autorotation is guaranteed by the following expression:

$\int_{\varphi_{1}}^{\varphi_{1}+2 \pi} \ddot{\varphi}(\varphi, \dot{\varphi}(\varphi)) d \varphi=0$

A similar equation was concluded by Klimina et al [17]. Suppose that $\varphi_{1}=0$ and $\dot{\varphi}=$ $\Phi|\sin \varphi|$, then applying equation 27 on equation 14 yields the following expression:

$I^{*}=3.183 \mu_{2} \frac{C \Phi}{V}$

This equation represents a bifurcation curve which separates fluttering region from autorotation that is called separatrix. As can be seen, the separatrix depends on $\mu_{2}$ (nonlinear damping coefficient) and $C \Phi / V$ (the maximum tip speed ratio of the rotating plate). Bifurcation diagrams are presented in Figure 11 for experimental data and results of RFA model when $\mu_{2}=0.03$. As can be observed from diagrams, I* correspondent to bifurcation is almost independent on Reynolds number. The similar conclusion was reported by other researchers mostly by the experiment, e.g. in [26], [32], [33], etc. According to these figures, the bifurcated $I^{*}$ for experimental data is averagely 0.13 , a slightly change happens in the different Reynolds numbers, and for the analytical model is almost 0.14 . For I*s less than the bifurcated $\mathrm{I}^{*}$, the rotation of the plate is chaotic rotation (consists of the fluttering and the autorotation) or pure fluttering. Using equation 28 and also the bifurcated $I^{*}$ s, it can be stipulated that the maximum tip speed ratio of an autorotating plate is averagely 1.5, However, it is affected slightly by the changing of $\mu_{2}$. 

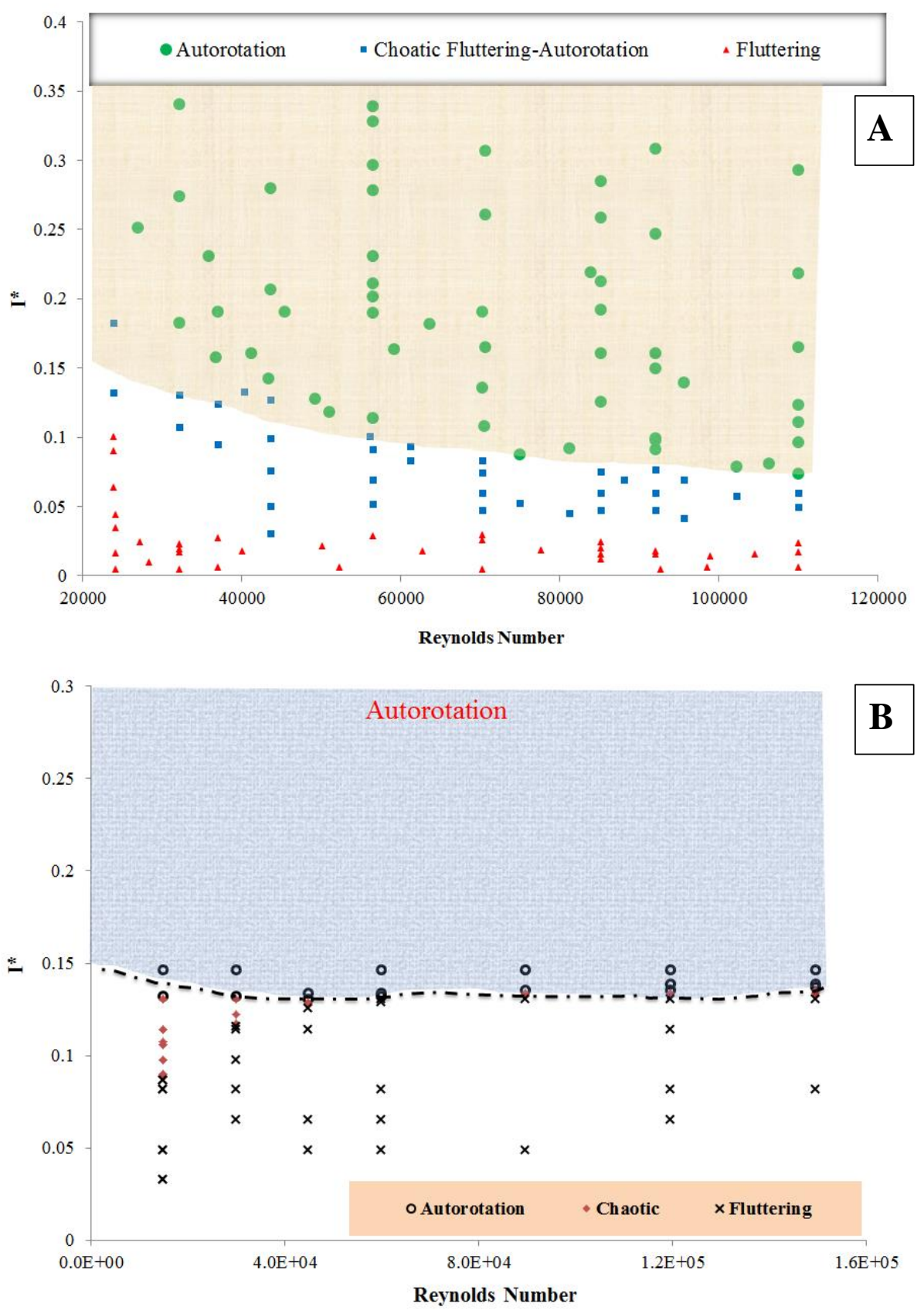

Figure 11: Bifurcation diagrams of I* versus Reynolds number A: Experimental data [34], B: results of $\operatorname{RFA}$ model $\left(\mu_{2}=0.03\right)$.

\section{Conclusion}

This paper was devoted to study on mathematical model of the fluttering and the autorotation of an articulated plate into the fluid flow. The fluttering is referred to oscillatory rotation of an object into the current and the autorotation is pointed out to a continuous rotation without any external power. The phenomena of fluttering and autorotation of the plate will occur when the moment of inertia reaches the appropriate criterion. In other words, transition from fluttering to tumbling for an object happens in large enough moment of inertia that is indicated by dimensionless number of $I^{*}$. $I^{*}$ is defined as ratio of the moment of inertia of the object to its added moment of inertia. 
Two mathematical models related to a falling object, reported by Belmonte et al [20] and Pessavento et al [19], were considered in this study to generate two mathematical models for a hinged plate with 1 degree of freedom (1DOF). The generated models were called the RFA model and the RFB model. These models were solved numerically and compared to experimental data. It can be deduced from this study that the RFA is more appropriate than the RFB for simulating of all modes of rotation of an articulated plate into the fluid flow. These modes are the fluttering, chaotic and autorotation.

Because of the nonlinear dynamics of an articulated plate into the flow, the stability and bifurcation analysis have been carried out on the RFA model. By means of such analysis, it was found that this model has a transcritical bifurcation and therefore stability diagram and threshold have been presented. In addition, an analytical expression was given for finding the boundary of bifurcation of the fluttering and the autorotation. In this respect, a bifurcation diagram (I* versus velocity) was presented by the numerical calculation on the RFA. Based on this diagram, the bifurcation boundary is approximately independent of the velocity and only depends on damping terms.

\section{Appendix A}

This section explains how a RFB model is derived from Belmonte equations (Equation 3). The same procedure is used to obtain the RFA model from Equation 9.

Equation 3-a represents the force in direction of $x$ and therefore it generates no torque on the plate. On the other side, equation 3-b calculates the perpendicular force to the plate at the center of pressure (cp), therefore it can produce a torque when is multiplied by Xcp $(f)$.

$f \times\left[\dot{v}_{y}+v_{x} \dot{\varphi}\right]=f \times\left[\frac{1}{C F r^{2}}\left[-\delta_{\perp} V v_{y}-4 \pi v_{x} v_{y} \operatorname{Sgn}\left(v_{x}\right)\right]\right]$

Equation 3-c represents the torque, as well. Hence, the equation is obtained by accumulating the torque from the equation 1-A and equation 3-c in which insert the values of velocities (i.e. $v_{x}$ and $v_{y}$ ) and accelerations (i.e. $\dot{v}_{x}$ and $\dot{v}_{y}$ ) from equation 10 and 12.

$$
\begin{aligned}
& f V \dot{\varphi} \sin \varphi-f \dot{f} C \dot{\varphi}-f^{2} C \ddot{\varphi}+f v_{x} \dot{\varphi}=\frac{\delta_{\perp} f V^{2} \cos \varphi}{C F r^{2}}+\frac{\delta_{\perp} V f^{2} \dot{\varphi}}{F r^{2}}+\frac{4 \pi f V^{2} \sin \varphi \cos \varphi \operatorname{sgn}\left(v_{x}\right)}{C F r^{2}}+\frac{4 \pi V f^{2} \sin \varphi \operatorname{sgn}\left(v_{x}\right)}{F r^{2}} \quad 2-A \\
& \ddot{\varphi}=-12 \frac{\delta_{\omega}|\dot{\varphi}| \dot{\varphi}}{F r^{2}}+\frac{12 \pi}{C^{2} F r^{2}} V^{2} \sin \varphi \cos \varphi+\frac{12 \pi}{C F r^{2}} f V \dot{\varphi} \sin \varphi
\end{aligned}
$$

Now, Equation 13 results from (2-A) + (3-A). Note that the values of $f^{2}$ and $f \dot{f}$ are small with respect to $\mathrm{C}$ and therefore are negligible.

\section{Acknowledgement}

The authors acknowledge greatly CNPq (The Brazilian National Research Council), and LOC/COPPE/UFRJ (Laboratory of Waves and Current of COPPE, Federal University of Rio de Janeiro).

\section{References}

[1] Fernandes, A. C., Rostami, A. B., Canzian, L. G., \& Sefat, S. M. (2013, June). Vertical Axis Current Turbine (VACT) and Its Efficiency. In Proceedings of the ASME 2013 32nd 
International Conference on Ocean, Offshore and Arctic Engineering, Nantes, France, OMAE2014 , pp. V008T09A051-V008T09A051.

[2] Armandei, M., Fernandes, A. C. \& Rostami A.B. (2016) Hydroelastic Buffeting Assessment Over a Vertically Hinged Flat Plate. Experimental Techniques, 40(2), 833-839.

[3] Rostami, A. B., \& Armandei, M. (2017). Renewable energy harvesting by vortex-induced motions: Review and benchmarking of technologies. Renewable and Sustainable Energy Reviews, 70, 193-214.

[4] Fernandes, A. C., \& Armandei, M. (2014, June). Van Der Pol-Duffing Modeling for Torsional Galloping. In ASME 2014 33rd International Conference on Ocean, Offshore and Arctic Engineering (pp. V09BT09A009-V09BT09A009). American Society of Mechanical Engineers.

[5] Fernandes, A. C. \& Armandei, M. (2011, January). Passive energy extraction through oscillation of a yawing flat plate operating in a uniform current. In ASME 2011 30th International Conference on Ocean, Offshore and Arctic Engineering (pp. 879-886). American Society of Mechanical Engineers.

[6] Bakhshandeh rostami, A., (December 2015). Energy Harvesting by Hydro/Aero Elastic Phenomena In Small Scale, In R. Williams (Ed.) Energy Harvesting, Technology, Methods and Application, Ed. 1, Chapter 4, (pp.83-110). New York, NY: Nova Science Publishers.

[7] Fernandes, A. C. \& Armandei, M. (2013, May). Marine Current Energy Extraction Using Torsional Galloping Based Turbine. In Offshore Technology Conference. Offshore Technology Conference.

[8] Rostami, A. B., \& Fernandes, A. C. (2014, June). Plate shape effect on the performance of the vertical axis auto rotation current turbine (VAACT). InASME 2014 33rd International Conference on Ocean, Offshore and Arctic Engineering (pp. V09AT09A041V09AT09A041). American Society of Mechanical Engineers.

[9] Fernandes, A. C. \& Rostami A.B. (2015). Hydrokinetic energy harvesting by an innovative vertical axis current turbine. Renewable Energy, 81, 694-706.

[10] Rostami, A. B., \& Fernandes, A. C. (2015). The effect of inertia and flap on autorotation applied for hydrokinetic energy harvesting. Applied Energy, 143, 312-323.

[11] Ghadimi, P. Rostami, A.B. \& Jafarkazemi, F. (2012) Aerodynamic Analysis of the Boundary Layer Region of Symmetric Airfoils at Ground Proximity. Aerospace Science and Technology, 17(1), 7-20.

[12] Zeraatgar, H., Rostami, A.B., \& Nazari, A. (2012). A Study on Performance of PlaningWing Hybrid Craft. Polish Maritime Research, 19(4), 16-22.

[13] Lugt, H. J. (1983). Autorotation. Annual Review of Fluid Mechanics, 15(1), 123-147.

[14] Pandula, Z., Kullmann, L., \& Hos, C. (2002). Analysis of the free-fall of a disc in viscous fluid using the techniques of nonlinear dynamics. 
[15] Borisov, A. V., Kuznetsov, S. P., Mamaev, I. S., \& Tenenev, V. A. (2016). Describing the motion of a body with an elliptical cross section in a viscous uncompressible fluid by model equations reconstructed from data processing. Technical Physics Letters, 42(9), 886890.

[16] Copeland, G. S. (1994). A perturbed-pendulum model for flat-plate autorotation. Journal of fluids and structures, 8(2), 125-138.

[17] Klimina, L., Lokshin, B., \& Samsonov, V. (2009). Parametrical Analysis of the behavior of an Aerodynamic Pendulum with Vertical Axis of Rotation. InModeling, Simulation and Control of Nonlinear Engineering Dynamical Systems (pp. 211-220). Springer Netherlands.

[18] Lugt, H. J. (1980). Autorotation of an elliptic cylinder about an axis perpendicular to the flow. Journal of Fluid Mechanics, 99(04), 817-840.

[19] Andersen, A., Pesavento, U., \& Wang, Z. (2005). Analysis of transitions between fluttering, tumbling and steady descent of falling cards. Journal of Fluid Mechanics, 541, 91104.

[20] Belmonte, A., Eisengerg, H. \& Moses, E., (1998). From flutter to tumble: inertial drag and Froude similarity in falling paper. Physical Review Letters,81(2), 345.

[21] Skews, B. W. (1990). Autorotation of rectangular plates. Journal of Fluid Mechanics, 217, 33-40.

[22] Iversen J.D., 1979, "Auto-rotating flat plate wings: the effect of moment of inertia, geometry and Reynolds number”, J. fluid Mechanics, Vol. 92, part 2, pp. 327-348.

[23] Smith, E. H. (1971). Autorotating wings: an experimental investigation. Journal of Fluid Mechanics, 50(03), 513-534.

[24] Rostami, A. B., Ghadimi, P., \& Ghasemi, H. (2016). Adaptive viscous-inviscid interaction method for analysis of airfoils in ground effect. Journal of the Brazilian Society of Mechanical Sciences and Engineering, 38(6), 1593-1607.

[25] Kuznetsov, S. P. (2015). Plate falling in a fluid: Regular and chaotic dynamics of finitedimensional models. Regular and Chaotic Dynamics, 20(3), 345-382.

[26] Willmarth, W. W., Hawk, N. E., \& Harvey, R. L. (1964). Steady and Unsteady Motions and Wakes of Freely Falling Disks. Physics of Fluids, 7, 197-208.

[27] Wick H.B. 1954, "Study of the subsonic forces and moments on an inclined plate of infinite span”, NACA TN 3221.

[28] Ghadimi, P., Bandari, H. P., \& Rostami, A. B. (2012). Determination of the heave and pitch motions of a floating cylinder by analytical solution of its diffraction problem and examination of the effects of geometric parameters on its dynamics in regular waves. International Journal of Applied Mathematical Research, 1(4), 611-633.

[29] Salehi, M., Ghadimi, P., \& Rostami, A. B. (2014). A more robust multiparameter conformal mapping method for geometry generation of any arbitrary ship section. Journal of Engineering Mathematics, 89(1), 113-136. 
[30] Zeraatgar, H., \& Rostami, A. B. (2012). An investigation on ship operability versus equipment operability in irregular waves. Brodogradnja, 63(1), 30-34.

[31] Hargreaves, D. M., Kakimpa, B., \& Owen, J. S. (2014). The computational fluid dynamics modelling of the autorotation of square, flat plates. Journal of Fluids and Structures, 46, 111-133.

[32] Field S. B., Klaus M., Moore M. G., Nori F., 1997, "Chaotic dynamics of falling disks", Nature, Vol. 388, pp 252-254.

[33] Smith E. H., 1971 “Autorotating wings: an experimental investigation” Journal of Fluid Mechanics, vol. 50, pp. 513-534.

[34] Bakhshandeh rostami, A. (2016). Hydrokinetic energy harvesting by autorotation of a plate with hinged axis ( $\mathrm{PhD}$ thesis, UFRJ). 